\title{
Correction to: New insights into the relationship between seismic intensity measures and nonlinear structural response
}

\author{
Yeudy F. Vargas-Alzate ${ }^{1}$ (i) . Jorge E. Hurtado ${ }^{2} \cdot$ Luis G. Pujades $^{1}$
}

Published online: 1 February 2022

(c) Springer Nature B.V. 2022

\section{Correction to: Bulletin of Earthquake Engineering} https://doi.org/10.1007/s10518-021-01283-x

This corrections is published as vendor overlooked several corrections related with Table 2 . Original article has been updated.

Publisher's Note Springer Nature remains neutral with regard to jurisdictional claims in published maps and institutional affiliations.

The original article can be found online at https://doi.org/10.1007/s10518-021-01283-X.

Yeudy F. Vargas-Alzate

yeudy.felipe.vargas@upc.edu

1 Departament d'Enginyeria Civil i Ambiental, Universitat Politècnica de Catalunya BarcelonaTech (UPC), Barcelona, Spain

2 Departamento de Ingeniería Civil, Universidad Nacional de Colombia, Manizales, Colombia 